\title{
Fatores associados ao sobrepeso e obesidade infantil
}

\author{
Factors associated with child overweight and obesity \\ Factores asociados con el niño sobrepeso y obesidad
}

Lourena de Melo Almeida ${ }^{1 *}$, Walnara Arnaud Moura Formiga², Rafael Ferreira Lima ${ }^{2}$, Waleska Gualberto da Silva1, Izabela Letícia de Andrade e Silva1 ${ }^{1}$, Sabrina Bezerra da Silva², Íngrid Raquel Martins Gomes Fernandes ${ }^{1}$, Amanda de França Ramos ${ }^{1}$, Thiago Alves Viana ${ }^{1}$, Érika Maria Gomes de Araújo Nóbrega ${ }^{3}$.

\section{RESUMO}

Objetivo: O estudo teve como objetivo investigar a influência de fatores associados ao sobrepeso e obesidade infantil. Revisão bibliográfica: Diante do levantamento de dados foi possível identificar que a taxa de sobrepeso e obesidade no período é um fator importante no impacto da saúde mundial, uma vez que nesse período são definidos os hábitos alimentares que servirão como norte para atitudes alimentares na fase adulta. Nessa fase que são construídas as escolhas alimentares e com isso a definição do estado nutricional do indivíduo adulto, com isso é crucial a prática de hábitos alimentares saudáveis afim de reduzir o número de doenças crônicas não-transmissíveis, sendo uma delas e a que mais necessita de atenção a obesidade. Os índices de obesidade no mundo são alarmantes e com isso caracteriza-se um problema de saúde pública, necessitando intervenção de políticas de saúde. Considerações finais: É evidente a importância da educação nutricional e alimentar nesse período da vida, pois a partir desses, atitudes alimentares saudáveis serão instaladas e maiores índices de adultos saudáveis.

Palavras-chave: Epidemiologia, Nutrição infantil, Doenças crônicas não transmissíveis.

\section{ABSTRACT}

Objective: The study aimed to investigate the influence of factors associated with overweight and childhood obesity. Literature review: Before the data collection was identified that the rate of overweight and obesity in the period is an important factor in the impact of global health, since that period eating habits are established that will serve as north to eating attitudes in adulthood. At this stage, food choices are built and, thus, the definition of the nutritional status of the adult individual, thus it is crucial to practice healthy eating habits in order to reduce the number of chronic non-communicable diseases, one of which is the one that most needs it attention to obesity. The obesity rates in the world are alarming and with that a public health problem is characterized, requiring the intervention of health policies. Final thoughts: It is evident the importance of nutritional and food education in this period of life, as from these, healthy eating attitudes will be installed and higher rates of healthy adults.

Keywords: Epidemiology, Child nutrition, Chronic non-communicable diseases.

\section{RESUMEN}

Objetivo: El estudio tuvo como objetivo investigar la influencia de los factores asociados con el sobrepeso y la obesidad infantil. Revisión bibliográfica: En vista de la encuesta de datos, fue posible identificar que la tasa de sobrepeso y obesidad en el período es un factor importante en el impacto de la salud global, ya que en este período se definen los hábitos alimenticios que servirán como guía para las actitudes alimentarias en

${ }^{1}$ Centro Universitário de Patos (UNIFIP), Patos - PB, *E-mail: lourena_melopb@hotmail.com

2 Universidade Federal de Campina Grande (UFCG), Pombal - PB.

${ }^{3}$ Universidade Federal da Paraíba (UFPB), João Pessoa - PB. 
la edad adulta. En esta etapa, se construyen elecciones de alimentos y, por lo tanto, la definición del estado nutricional del individuo adulto, por lo que es crucial practicar hábitos alimenticios saludables para reducir la cantidad de enfermedades crónicas no transmisibles, una de las cuales es la que más lo necesita. Atención a la obesidad. Las tasas de obesidad en el mundo son alarmantes y con eso se caracteriza un problema de salud pública que requiere la intervención de políticas de salud. Consideraciones finales: Es evidente la importancia de la educación nutricional y alimentaria en este período de la vida, ya que a partir de estos, se instalarán actitudes de alimentación saludable y mayores tasas de adultos sanos.

Palabras clave: Epidemiología, Nutrición infantil, Enfermedades crónicas no transmisibles.

\section{INTRODUÇÃO}

A obesidade é uma doença multifatorial caracterizada pelo acúmulo excessivo de gordura corporal, que interfere na expectativa e qualidade de vida do indivíduo. Pode ser causada por fatores nutricionais, genéticos, psicossociais, culturais, entre outros. Está associada a riscos de desenvolver doenças crônicas não transmissíveis, tais como diabetes, hipertensão arterial e doenças cardiovasculares (CARVALHO KMB e DUTRA ES, 2014; BARBOSA JM e NEVES CMAF, 2013).

Segundo Barbosa JM e Neves CMAF (2013), os fatores genéticos exercem influência no ganho de peso de indivíduos, o estilo de vida e as escolhas alimentares são os principais responsáveis. Os hábitos alimentares e de atividades física são obtidos na infância, quando esses se encontram inadequados tem como consequência o sobrepeso e a obesidade. A falta de exercício, o tempo excessivo gasto com o uso de aparelhos eletrônicos e o consumo de alimentos industrializados são os principais fatores ambientais que contribuem para o aumento de peso excessivo em crianças.

O número global de crianças e adolescentes obesas ou acima do peso aumentou para 42 milhões em 2013. Se as tendências atuais continuarem, o número de crianças com excesso de peso em todo o mundo aumentará para 70 milhões até 2025 (OMS, 2015). Em concordância com Monteiro JP et al. (2013), quando crianças que possuem genética suscetível estão em ambiente que promove comportamento que favoreça a obesidade, o aumento de peso é comprovado.

Conforme Barbosa JC, et al. (2015), o sobrepeso e a obesidade são graves problemas de saúde pública, sendo encontradas altas prevalências desse distúrbio em escolares. Devido à obesidade ser uma doença crônica, a atenção em relação aos hábitos de vida dessa população deve ser de caráter permanente.

Outros fatores associados à obesidade infantil são apontados por Melzer MRTF, et al. (2015), onde identificaram uma relação significativa entre o acúmulo de gordura e o excesso de peso da criança e acúmulo de gordura abdominal materno, constatando então que o estado nutricional materno está associado ao da criança, independentemente do nível socioeconômico.

Ferrari GLM, et al. (2015), identificaram em seu estudo que crianças estão gastando mais tempo em atividades sedentárias em contraposição a atividades físicas. A presença de equipamentos eletrônicos está relacionada negativamente a prática de atividade física. Além disso, a presença desses aparelhos foi associada com o Índice de Massa Corporal (IMC), onde crianças que não tinham equipamentos eletrônicos no quarto tinham menor média de IMC do que aquelas que tinham.

A relevância do tema sobrepeso/obesidade infantil como problema de saúde pública mundial ocorre por ser ao mesmo tempo uma doença e um fator de risco para as doenças crônicas não transmissíveis e está associada a um estilo de vida sedentário, assim como a hábitos alimentares incorretos influenciados pelo meio em que a criança está inserida e as condições socioeconômicas da família.

Diante deste fato, o diagnóstico precoce bem como a promoção de atividades de educação nutricional adequadas, tanto com a criança quanto com os familiares, são fundamentais para a inversão desse quadro e prevenção de futuros agravos à saúde da criança, já que a maioria delas apresenta excesso de peso devido aos hábitos alimentares errados bem como o sedentarismo, levando em consideração que as crianças são reflexos dos pais e comem o que eles ofertam. 
Assim sendo, a presente pesquisa se justifica nesse contexto, na tentativa de evidenciar quais fatores exercem maior influência com relação ao excesso de peso de crianças. Visto isso, o presente trabalho tem como objetivo investigar a influência de fatores associados ao sobrepeso e obesidade infantil.

\section{REVISÃO BIBLIOGRÁFICA}

\section{Obesidade}

Segundo a Organização Mundial da Saúde (2013), atualmente a obesidade é um dos problemas de saúde pública mais grave do século XXI e afeta todos os países em graus variados, principalmente os grupos socioeconômicos mais baixos. Estima-se que mais de $75 \%$ das crianças com sobrepeso vivem em países em desenvolvimento. Esta patologia está relacionada a diversos fatores, como hábitos alimentares, inatividade física, fatores biológicos, psicológicos e comportamentais.

Pesquisa realizada pelo Ministério da Saúde revela que o percentual de pessoas com excesso de peso supera mais da metade da população brasileira. A pesquisa mostra que $51 \%$ da população estão acima do peso e $17 \%$ da população encontram-se obesas. Isso é preocupante, já que a obesidade se constitui como um fator de risco para o desenvolvimento de outras doenças crônicas não transmissíveis (BRASIL, 2013).

O sobrepeso e a obesidade infantil vêm ganhando aumentos significativos em todo o mundo desde a década de 1990 e têm atingido indivíduos em fases de vida cada vez mais precoces da vida, sobretudo em países em desenvolvimento, onde se nota maiores índices (BARBOSA BB, et al., 2018).

Segundo dados da OMS (2016), globalmente existiam cerca de 40 milhões de crianças com menos de cinco anos com diagnóstico de pré-obesidade ou eram obesas no ano de 2012. Nessa mesma publicação evidenciou-se que esse número subiu para 41 milhões no ano de 2014.

O Brasil tem vivenciado uma rápida transição nutricional, de um país que apresentava altas taxas de desnutrição a um país com metade da população adulta com excesso de peso. Em virtude de sua magnitude e velocidade de evolução, o sobrepeso e a obesidade são considerados atualmente um dos problemas de saúde pública mais preocupante, afetando todas as faixas etárias (BRASIL, 2016).

De acordo com Carvalho KMB e Dutra ES (2014), há uma diferença sutil entre obesidade e sobrepeso, a primeira é uma doença crônica multifatorial caracterizada pelo excessivo acúmulo de gordura corporal, enquanto que o sobrepeso se caracteriza pelo aumento exclusivo de massa corporal, porém ambos acarretam em prejuízos sérios à saúde do indivíduo.

No caso da obesidade, suas repercussões clínicas dependem principalmente de onde está distribuído o excesso de gordura. A distribuição de gordura localizada nos quadris está associada com um risco maior de desenvolver artroses e varizes e é denominada de obesidade periférica ou ginóide. Quando a maior parte da gordura concentrada é visceral há um maior risco de desenvolvimento de doenças cardiovasculares, esta é denominada obesidade central ou androide.

A sociedade está vivenciando um mundo repleto de mudanças com foco na facilidade das atividades diárias e a falta de tempo para a prática de exercícios, assim como para uma alimentação saudável. Com tudo isso tem sido evidenciado um apelo social e científico a respeito da importância da intervenção baseada em hábitos de vida saudáveis, principalmente na saúde infantil, com foco na obesidade (ANDRADE J, et al., 2015).

A obesidade infantil está associada à possibilidade de sua manutenção ao longo da vida, aumentando a morbimortalidade principalmente na fase adulta, devido ao acúmulo excessivo de gordura corporal, tendo como consequências problemas cardiovasculares, respiratórios, endocrinológicos, psicossociais, dentre outros (MONTEIRO JP, et al., 2013).

Ainda são desconhecidos os diferentes impactos dos comportamentos alimentares e de atividade física nesse novo cenário da obesidade no Brasil. Como os hábitos alimentares e de prática de exercícios adquiridos na infância e na adolescência tendem a continuar na vida adulta, é grande importância a identificação das práticas não saudáveis e as suas causas para que políticas e programas de saúde sejam implementados visando a um melhor controle das patologias associadas na vida adulta (CARREGOSA WV, et al., 2015). 
Em conformidade com Andrade J, et al. (2015), a melhor forma de controlar a obesidade infantil baseia-se na prevenção e proteção à saúde, por meio de programas de educação nutricional, de práticas de atividades físicas e de conscientização de práticas saudáveis, sendo a escola e a família os principais condutores deste processo.

A participação dos pais no tratamento e no acompanhamento da obesidade de seus filhos é crucial para o sucesso das intervenções, o acompanhamento familiar necessita da promoção de hábitos saudáveis e apoio para lidar com as repercussões negativas da obesidade. Enfatiza-se a importância da sensibilização e a conscientização da família, assim como a participação da escola e da sociedade no incentivo da promoção da saúde, como alimentação adequada e práticas de exercício físico (VITORINO SVZ, et al., 2015; LIRA RCM, et al., 2016). Os comportamentos relacionados à saúde das crianças permaneçam sob a influência e o controle dos seus pais, enfrentar a epidemia da obesidade infantil se mostra difícil e é necessário, portanto, aprimorar o entendimento das consequências desse problema de saúde entre os pais (APARícIO G, et al., 2011).

Um desafio vital para a família de crianças obesas é proporcionar apoio e proteger a autoestima de seus filhos, ao mesmo tempo que se orienta adequadamente quanto às práticas alimentares mais apropriadas, notando uma necessidade de entender o indivíduo por completo para assim desenvolver um plano terapêutico efetivo para cada criança, adequando as necessidade dela e de todos envolvidos no contexto familiar (MELO VLC, et al., 2010).

\section{Avaliação Nutricional em crianças com excesso de peso}

Segundo Barbosa JM e Neves CMAF (2013), o diagnóstico de sobrepeso e obesidade em crianças é fundamentalmente clínico, tomando como base os dados antropométricos, no exame físico e na história clínica e nutricional. O Índice de Massa Corporal (IMC) é o indicador antropométrico mais simples utilizado para avaliação nutricional, principalmente para diagnóstico de excesso de peso e magreza em crianças e é recomendado internacionalmente para o diagnóstico individual ou coletivo de distúrbios nutricionais. Em crianças o IMC é classificado de acordo com a idade (MUSSOI DT, 2015).

Fontes GAV, et al. (2012), afirmam que o Índice de Massa Corporal/Idade (IMC/I) expressa a relação entre o peso da criança e o quadrado da estatura. Esse indicador acrescenta a informação da idade do indivíduo, então é recomendado para o diagnóstico nutricional de crianças, porém não fornece a composição corporal, sendo assim o ideal é que seja associado a outros indicadores.

Segundo Mussoi DT (2015), a localização da gordura corporal pode ser mais relevante do que a quantidade de gordura corporal total. A circunferência da cintura em crianças está associada a um maior risco de desenvolver alterações fisiopatológicas, tornando assim esta medida antropométrica ideal para associá-la ao IMC.

\section{Influência da alimentação no desenvolvimento da obesidade}

A indústria de alimentos foi, em grande parte, responsável pela mudança radical que se operou na alimentação nos últimos anos. O resultado é que cresceu de forma radical o número de pessoas obesas nas últimas décadas. O comércio de alimentos industrializados na porta da escola deve ser monitorado, considerando que a presença de vendedores desses gêneros pode ser um fator que irá contribuir negativamente nas práticas alimentares saudáveis dessas crianças (ARRUDA CAM, et al., 2015).

A formação dos hábitos alimentares saudáveis na infância é de grande relevância, devido à possibilidade de continuidade na vida adulta desses hábitos, isso vai influenciar diretamente no estado nutricional. Devido a mudança no perfil nutricional que vem ocorrendo nos últimos anos e com a formação de hábitos alimentares inadequados, a obesidade infantil, bem como o risco de desenvolvê-la vem aumentando consideravelmente (PORTO ACV, et al., 2013).

Moreira MSF, et al. (2014), afirmam que o aparecimento dos problemas de saúde na infância, relacionadas com a ingestão energética, como no caso da obesidade, estão frequentemente associadas ao desequilíbrio entre a atividade física e o consumo alimentar. A obesidade na infância é mais difícil do que na idade adulta, devido ao fato de que criança não tem entendimento quanto aos danos causados por esta patologia. 
Barros MP (2015), atesta que é notável o crescimento do consumo de produtos industrializados e sua interferência nos hábitos alimentares das famílias. Desta maneira é imprescindível refletir sobre a questão da publicidade feita para promover alimentos não saudáveis dirigidos ao público infantil e sua responsabilidade, visto que hábitos alimentares são formados por cada indivíduo desde muito cedo e seguidos ao decorrer da vida. O consumo excessivo de energia proveniente de gorduras e açúcares em contraposição de um menor consumo de frutas e verduras são um dos principais fatores associado à obesidade infantil (BARBOSA JM e NEVES CMAF, 2013).

Estudo realizado por Garcia C, et al. (2014), atestou-se que as crianças que apresentavam sobrepeso e obesidade consumiam frequentemente alimentos industrializados que são ricos em gordura, açúcar e sódio, como exemplos os refrigerantes, salgadinhos de pacote, biscoitos recheados, guloseimas, doces, dentre outros. Alimentos muito calóricos os quais quando consumidos de maneira regular e exagerados podem ocasionar doenças crônicas não transmissíveis como hipertensão arterial sistêmica, diabetes mellitus, dislipidemias e doenças cardiovasculares.

De acordo com Porto ACV, et al. (2013), o consumo excessivo de alimentos ricos em calorias e do tipo fast food, seja por influência de amigos, família ou mídia, fazem com que as crianças iniciem cedo um hábito alimentar incorreto e consequentemente, deixem de conhecer alimentos necessários, presentes nos grupos de alimentos que devem ser consumidos diariamente.

Cruz MJSMA (2014), sugere que os hábitos alimentares, assim como, os comportamentos sedentários, possam ser influenciados positivamente com a execução de programas educacionais em infantis até aos 12 anos. A eficácia destas medidas educativas de intervenção depende de um maior conhecimento dos fatores influenciadores e mediadores destes comportamentos nesta fase da vida.

\section{Relação entre o estado nutricional da criança e o ambiente familiar}

A criança está inserida em um ambiente onde os hábitos alimentares estão se transformando, onde os produtos industrializados estão facilmente acessíveis, e os pais não dispõem de tempo para adquirir alimentos mais saudáveis e muito menos para o preparo dos mesmos em casa (BARROS MP, 2015).

Em estudo realizado por Porto ACV, et al. (2013), constatou-se que há influência do ambiente familiar no ganho de peso das crianças. Sendo o cenário familiar um lugar de troca de carinhos, dedicação, hábitos e cultura, uma maior incidência de crianças com excesso de peso é constatado em contextos familiares pouco funcionais, podendo-se afirmar que $o$ ato de comer poderá ser para a criança, por um lado, a forma que encontra para se inserir em uma família onde se sente vazia de afetos e, por outro, uma maneira de usar o corpo como proteção de um ambiente familiar disfuncional.

De acordo com Ancona-Lopez F, et al. (2014), já que a obesidade, os excessos alimentares e a falta de atividade física são sintomas agravantes do emocional da criança, é preciso atentar-se a dinâmica e o ambiente familiar, o vínculo mãe-filho onde estão inseridas essas crianças.

Em estudo de Porto ACV, et al. (2013), atestou-se que as crianças que percebem um ambiente familiar onde se sentem apoiadas, em que há um maior envolvimento e liberdade para expressar os seus sentimentos, apresentam maior normalidade com relação ao peso. Sendo assim, uma maior funcionalidade familiar associou-se positivamente com hábitos alimentares mais saudáveis e a comportamentos que remetem para uma ingestão mais controlada.

Em conformidade com Rosaneli CF, et al. (2012), o crescimento da obesidade em crianças ocasiona um forte impacto econômico e em contrapartida uma diminuição na qualidade de vida. É imprescindível que os pais tenham conhecimento da importância do seu papel na prevenção e controle da obesidade infantil para que haja sucesso e suporte efetivo.

Segundo Dias CMSB e Morais PM (2013), a mudança de hábitos alimentares dos membros familiares devido às exigências do tratamento, os obstáculos que elas enfrentam para compreender a necessidade de estabelecer limites à criança e para solicitar a contribuição de todos são as dificuldades encontradas no ambiente familiar para a superação do problema. 


\section{Estado nutricional associado a fatores socioeconômicos}

De acordo com a Organização Mundial de Saúde (2016), as crianças vêm crescendo em ambientes que favorecem o excesso de peso, incentivados pela globalização e urbanização, exposição a ambientes insalubres que ocorrem em todos os grupos socioeconômicos. A facilidade com que se encontram alimentos e bebidas pouco saudáveis no mercado é um fator importante para o aumento do número de crianças com sobrepeso e obesidade, especialmente nos países em desenvolvimento.

Para Barbosa JC, et al. (2015), o contexto familiar com menor rentabilidade potencializa o desenvolvimento de obesidade geral e/ou centralizada em crianças. Nesse mesmo estudo, aponta-se que crianças da rede pública de ensino tiveram IMC, Circunferência da Cintura (CC) e Razão Cintura Estatura (RCEst) elevados de forma isolada.

Em estudo realizado por Arpini LSB, et al. (2015), mostrou que os determinantes socioeconômicos influenciaram diretamente na qualidade da alimentação dos escolares. Algumas das variáveis socioeconômicas que estiveram associadas à qualidade da dieta ingerida pelos escolares foram classe socioeconômica e escolaridade materna. As crianças das classes socioeconômicas média apresentaram, em média, $51 \%$ mais chances de baixo índice de qualidade da dieta. Os escolares cujas mães relataram menos do que três anos de estudo apresentaram o triplo de chances de apresentar baixa qualidade da dieta.

\section{Correlação entre obesidade e atividade física}

Segundo Paes ST, et al. (2014), a prática de exercícios físicos é capaz de promover reações positivas sobre a obesidade infantil e atuar como coadjuvante na sua prevenção e tratamento. A magnitude dos benefícios pode variar conforme o exercício físico. Os principais efeitos provenientes dos exercícios estão associados à restauração do perfil lipídico, melhoria da composição corporal, mobilização dos substratos energéticos e à ativação metabólica.

Ferrari GLM, et al. (2015) evidencia associações entre a presença de equipamentos eletrônicos no quarto com atividade física de moderada a vigorosa e Índice de Massa Corporal (IMC), independentemente do sexo, escola e renda familiar anual. Em particular, dois ou três equipamentos eletrônicos no quarto estão associadas com baixa AFMV (Atividade Física de Moderada a Vigorosa) e alto IMC.

De acordo com Andrade J, et al. (2015), crianças obesas após intervenção, em função da prática de atividade física e, consequentemente, da evidente redução de peso, passaram a ter mais disposição, fazendo com que a autoestima melhorasse. Ferrari GLM (2015), enfatiza que o professor de educação física tem os meios de se aproximar do aluno, propondo assim mudanças de hábitos que sejam divulgados e incorporados à família dos escolares, levando em consideração assim que a prevenção e promoção de hábitos saudáveis é o melhor caminho.

\section{CONSIDERAÇÕES FINAIS}

O levantamento de dados possibilitou o entendimento profundo acerca dos fatores associados ao sobrepeso e obesidade na infância. Dentre eles, os maiores determinantes são: o contexto familiar onde essa criança está inserida, as condições financeiras, o nível socioeducacional dos pais, e ainda o nível de atividade física dessas crianças. Sendo assim, é notório que hábitos saudáveis durante a infância reflete em adultos mais saudáveis, uma vez que esses hábitos instalados, são reproduzidos pelo resto da vida. É notório a importância do profissional nutricionista no contexto da educação alimentar e nutricional no contexto familiar desde os primeiros momentos da vida, assim como também em todos os ciclos de vida. Ainda, a partir dos dados levantados é perceptível que a temática abordada é de importância para a comunidade científica, uma vez que os dados atuais de epidemiologia mostram altos índices de sobrepeso e obesidade na fase da infância.

\section{REFERÊNCIAS}

1. ARPINI LSB, et al. Associação entre a percepção materna do estado nutricional do filho e a qualidade da dieta de escolares. DEMETRA: alimentação, nutrição e saúde, 2015;10(2): 299-314. 
2. ANCONA-LOPEZ F, et al. Problemas Psicológicos e Psicodinâmicos de Crianças e Adolescentes Obesos: Relato de Pesquisa. Psicologia: Ciência e profissão, 2014; 34(1): 126-141, 201.

3. ANDRADE J, et al. Intervenções escolares para redução da obesidade infantil: uma revisão sistemática. Ciência e Saúde, 2015; 1(1): 72-78.

4. APRícIO G, et al. Olhar dos pais sobre o estado nutricional das crianças pré-escolares. Millenium, 2011; 20(40): 99113.

5. ARRUDA CAM, et al. Percepção de pais e professores sobre a Influência dos alimentos industrializados a saúde infantil. Revista Brasileira de Promoção à Saúde, 2015; 28(1): 16-22.

6. BARBOSA BB, et al. Percepção de cuidadores de crianças obesas acerca da obesidade infantil. SANARE, 2018; 17(2): 49-55.

7. BARBOSA JC, et al. Prevalência de sobrepeso e obesidade em crianças de quatro escolas estaduais de Belo Horizonte, Minas Gerais. Revista Médica de Minas Gerais, 2015; 25(2): 180-186.

8. BARROS MP. A influência da publicidade de alimentos na obesidade infantil. Trabalho de Conclusão de Curso (Graduação em Comunicação Social) - Centro Universitário de Brasília, 2015.

9. BARBOSA JM e NEVES CMAF. Obesidade. In: BARBOSA, J. M. et al. Guia Ambulatorial de Nutrição Materno-infantil. p. 137-146. 1 ed. Rio de Janeiro: Medbook, 2013.

10. BRASIL. Ministério da Saúde. 2013. Mais da metade da população brasileira tem excesso de peso. Disponível em: < http://portalsaude.saude.gov.br/index.php/o-ministerio/principal/portal-dent/mais-sobre-portal-dcnt?start=10>. Acesso em 07 de maio 2020.

11. BRASIL. DataSUS. Estado Nutricional dos Usuários da Atenção Básica. 2016. Rio de Janeiro. Disponível em: <http://tabnet.datasus.gov.br/cgi-win/SISVAN/CNV/notas_sisvan.html> Acesso em 20 de abril 2020.

12. CARREGOSA WV, et al. Obesidade e sedentarismo: fatores de risco em escolares. VEPEEF. Pernambuco. 2015.

13. CARVALHO KMB e DUTRA ES. Obesidade. In: Cupparl, L. Nutrição Clínica no adulto. $3^{\circ}$ ed. São Paulo: Manole; $2014 ; 185-214$

14. CRUZ MJSMA. Obesidade infantil: relação com a alimentação, atividade física e comportamentos sedentários. Dissertação (Mestrado em Exercício e Bem-Estar) - Universidade Lusófona de Humanidades e Tecnologias, Lisboa, 2014.

15. DIAS CMSB, MORAES PM. Nem Só de Pão se Vive: A Voz das Mães na Obesidade Infantil. Psicologia: Ciência e Profissão, 2013; 33(1):46-59.

16. FERRARI GLM. Associação entre equipamentos eletrônicos no quarto com tempo sedentário, atividade física e índice de massa corporal de crianças. Jornal de Pediatria, 2015; 91(6): 574-582.

17. FONTES GAV, et al. Manual de avaliação nutricional e necessidade energética de crianças e adolescentes uma aplicação prática. EDUFBA. Bahia, 2013.

18. GARCIA C, et al. Consumo alimentar: um estudo sobre crianças com sobrepeso e obesidade do Espaço Mamãe Criança de Vera Cruz/RS. CINERGIS, 2014; 15(4): 195-200.

19. LIRA RCM, et al. Experiência de abordagem sobre alimentação entre crianças. SANARE, 2016; 15(1): 69-73.

20. MELZER MRTF, et al. Fatores associados ao acúmulo de gordura abdominal em crianças. Revista Paulista de Pediatria, 2015; 33(4): 437-444.

21. MELO VLC, et al. Obesidade infantil: impactos psicossociais. Rev Méd Minas Gerais, 2010; 20(3): 367-370.

22. MOREIRA MSF, et al. Doenças associadas à obesidade infantil. Revista Odontológica de Araçatuba, 2014; 35(1): 6066.

23. MONTARROYOS ECL, et al. Antropometria e sua importância na avaliação do estado nutricional de crianças escolares. Comunicação, Ciências e Saúde, 2013; 24(1):21-26.

24. MONTEIRO JP, et al. Programa de Educação Nutricional em grupo para crianças e adolescentes com obesidade ambulatorial. In: GARCIA, R. W. D.; MANCUSO, A. M. C. Nutrição e Metabolismo: Mudanças alimentares e educação nutricional. Rio de Janeiro: Guanabara Koogan, 2013. p. 287-311.

25. MUSSOI TD. Avaliação Nutricional da prática clínica: da gestação ao envelhecimento. 1 ed. Rio de Janeiro: Guanabra Koogan, 2015; 5-129.

26. ORGANIZAÇÃO MUNDIAL DE SAÚDE. Recomendações da OMS sobre cuidados pré-natais para experiência positiva na gravidez, 2016

27. ORGANIZAÇÃO MUNDIAL DE SAÚDE. Número de crianças com excesso de peso pode chegar a 70 milhões até 2025, alerta OMS. 2015. Disponível em: https://nacoesunidas.org/numero-de-criancas-com-excesso-de-peso-podechegar-a-70-milhoes-ate-2025-alerta-oms/. Acesso em 9 de maio 2020.

28. ORGANIZAÇÃO MUNDIAL DE SAÚDE. Ceensp: Muito além do peso (Obesidade Infantil). 2013. Disponível em: <http://www5.ensp.fiocruz.br/biblioteca/home/exibedetalhesBiblioteca.cfm?ID=16611\&tipo=B>. Acesso em 22 de abril 2020.

29. PAES ST, et al. Efeitos metabólicos do exercício físico na obesidade infantil: uma visão atual. Revista Paulista de Pediatria, 2015; 33(1): 122-129.

30. PORTO ACV, et al. Frequência de consumo de fast food em crianças de uma escola pública e uma escola privada do município de Nova Iguaçu no Rio de Janeiro e sua influência no perfil nutricional. Acta Pediátrica Portuguesa, 2013; 44(6): 301-305.

31. ROSANELI CF, et al. Avaliação da prevalência e de determinantes nutricionais e sociais do excesso de peso em uma população de escolares: análise transversal em 5.037 crianças. Revista Associação de Medicina Brasileira, 2012; 58(4): $472-476$.

32. VIROTINO SVZ, et al. Viver com obesidade infantil: a experiência de crianças inscritas em programa de acompanhamento multidisciplinar. Revista RENE, 2015; 15(6): 980-989. 